\title{
The Heavy Ion Fusion Program In the USA
}

\author{
Roger O. Bangerter \\ Lawrence Berkeley National Laboratory and \\ Virtual National Laboratory for Heavy Ion Fusion
}

\begin{abstract}
The U.S. Department of Energy has established a new, larger inertial fusion energy program. To manage program growth, we have developed a new inertial fusion energy research and we have establishes a Virtual National Laboratory for Heavy Ion Fusion.

There has been significant technical progress. Improvements in target design have reduced the predicted energy requirements by approximately a factor of two. There have also been important experiments on chamber dynamics and other inertial fusion technologies. The accelerator program has completed a number of small-scale experiments. Experiments with driver-scale beams are being designed -- including experiments with driver-scale ion sources and injectors.
\end{abstract}

Finally we are developing the technologies needed to build a major research facility known as the Integrated Research Experiment (IRE).

\section{Introduction}

Interest in inertial fusion energy (IFE) research in the USA has increased significantly during the last two years. The inertial fusion community has developed a new development plan with clear milestones leading to a demonstration power plant (IFE Demo). Figure 1 is a graphical representation of the new plan. In developing this plan, we started from the goal of an IFE Demo in approximately 2025 and worked backward to determine what was necessary to achieve this goal. The plan preceding the IFE Demo has four steps: Concept Exploration, Proof of Principle, Performance Extension, and Fusion Energy Development. There is a parallel plan for magnetic fusion that has the same four steps. For each step we adopted a cost goal. We based these cost goals on judgements about the funding environment in the USA and on the value of fusion relative to other long-term energy sources. There is a decision point at the end of each step. Figure 1 shows these decision points and their approximate dates. 


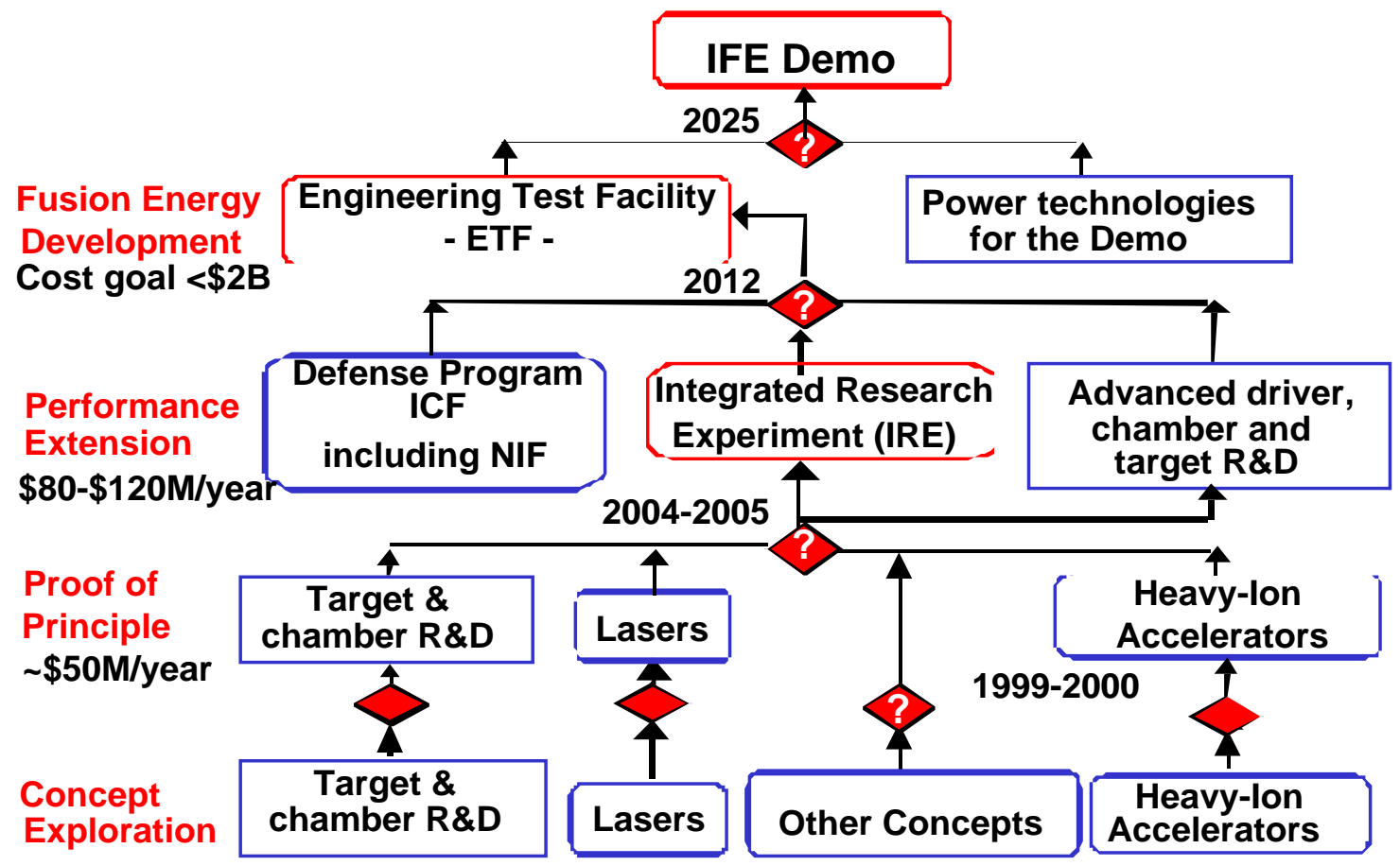

Figure 1 A graphical representation of the new national IFE program plan. Three IFE drivers have now advanced from the Concept Exploration level to the Proof of Principle (PoP) level. They are: heavy ion accelerators, KrF lasers, and diode pumped solid state lasers. PoP research on heavy ion accelerators has been in progress for about a year. This paper will discuss only the heavy ion fusion program. The goal of the PoP phase of the program is to obtain enough information to justify and build a so-called Integrated Research Experiment (IRE). The IRE together with the target physics information from the Department of Energy's Defense Inertial Confinement Fusion Program (ICF) and other target and chamber research programs, will provide the basis for the construction of an Engineering Test Facility (ETF). In the USA, the National Ignition Facility (NIF) is a cornerstone of the Defense ICF Program. Although the NIF has recently experienced some delays, it should nevertheless provide data in time for the decision to proceed with the ETF. The ETF will be the first IFE facility to produce high levels ( $>100 \mathrm{MW}$ ) of average fusion power. The ETF and continuing research on other fusion power technologies such as target fabrication and chambers will provide the basis for the Demo.

The PoP phase of the program is built on the theory, simulation, and experiments of the last two and a half decades. During this period, we performed a number of small experiments that, in a scaled sense, established the beam physics and demonstrated the beam manipulations needed for a full-scale driver. The largest experiments, e.g., the Single Beam Transport Experiment (SBTE) and the Multiple Beam Experiment with 4 Beams (MBE-4) were performed at Berkeley but there have also been important experiments at Brookhaven, Argonne, and Lawrence Livermore National Laboratories and at the University of Maryland. These experiments have shown that it is possible to produce, transport, and accelerate heavy ion beams with more than adequate brightness for fusion applications [1]. The scaled experiments have been in a driver-relevant parameter regime in terms of important 
dimensionless parameters such as phase advance and depressed phase advance per lattice period. These experiments typically transported and accelerated approximately $10 \mathrm{~mA}$ of current per beam. The corresponding current in a full-scale driver is expected to be of the order of $1 \mathrm{~A}$. Consequently, there are still important, unresolved issues associated with the absolute scale of the current. The one exception to this statement (in the USA) is the experiments with our 2-MeV injector, an injector that delivers approximately $1 \mathrm{~A}$ of beam current. Other issues associated with the focusing and transport of high-current beams in a fusion chamber environment also remain largely unexplored experimentally.

The PoP phase of the program consists of several important tasks. These include a greatly expanded research effort in chamber dynamics and target fabrication and an increased effort in target design and accelerator research. This increased effort requires increased funding. Both the Department of Energy and Congress have supported increased funding. The total funding for IFE research has increased from approximately \$7 M in FY1998 to nearly \$20 M in FY1999 and about \$28 M in FY2000. Institutional participation, particularly in the chamber and target fabrication areas, has increased markedly. In order to manage the increased funding and increased institutional participation efficiently, we have created a Virtual National Laboratory (VNL) for Heavy Ion Fusion. The term "virtual" refers to the fact that the VNL does not exist as a single set of buildings at a single site. Moreover the individual people working in the VNL remain employees of the laboratories that created the VNL. The VNL is a mechanism for coordinating heavy ion fusion research. It combines the three largest accelerator research programs (LBNL, LLNL, and PPPL) under a single management. Although they are not officially members of the VNL, there is important participation from SNLA, NRL, SLAC, the University of Maryland, the University of Missouri, MIT, Advanced Magnet Laboratory, Advanced Ceramics, Allied Signal, National Arnold, MRC, and General Atomics. Another virtual laboratory, the Virtual Laboratory for Technology (VLT), manages the research in the chamber and target fabrication areas. The VLT also manages fusion technology research. VLT inertial fusion participants include LANL, INEEL, UCB, UCLA, UCSD, LLNL, Georgia Tech, General Atomics, and the University of Wisconsin. Since many of these institutions are also involved in magnetic fusion research, there is now greatly improved cooperation and understanding between magnetic and inertial fusion.

\section{Recent Progress}

The increased funding and increased institutional participation in the program have enabled significant progress. This section will outline recent progress in target design, chamber dynamics, and accelerators. Section III outlines the goals of the IRE and discusses several IRE prerequisites that must be accomplished in the PoP phase of the program.

Target Design. The target designs by Tabak and Callahan-Miller presented at the Heidelberg Symposium required $6 \mathrm{MJ}$ of beam energy to produce a target gain of approximately 60 . More recent designs, according to 2-dimensional Lasnex calculations, require 1 to $3 \mathrm{MJ}$ of beam energy and have predicted gain between 90 and 130[2]. The improvements in energy and gain have been obtained by improving the target coupling by reducing the size of the hohlraum relative to the size of the capsule. While this strategy is effective from a target standpoint, it requires small focal spot sizes leading to increased demands on beam brightness and focusing systems. Efforts are now underway to design targets that allow larger focal spots, but it is likely that smaller focal spots will always lead to smaller energy requirements and larger gain - at least for hohlraum targets.

Chamber Research and Other Associated Technologies. Recent work on chambers for heavy ion fusion has emphasized thick liquid wall protection. Thick liquid jets between the target and the first structural wall serve three functions: (1) They protect the wall from target 
products such as photons, charged particles, debris, and neutrons. (2) They contain lithium to breed the tritium needed for the targets. (3) They serve as the primary coolant. Target products other than neutrons can be stopped with relatively little material so the main advantage of thick liquids is their ability to attenuate the neutrons by large factors. This attenuation greatly reduces the activation of the structure and greatly increases its useful life. Despite these advantages there are important issues. The HYLIFE II chamber design [3] uses oscillating fluid jets to form a pocket or cavity for the target . It also uses crossed jets to protect the conductors of the final focusing elements. For both applications it is desirable to have jets with smooth surfaces. A significant number of droplets or spray associated with the jets could interfere with beam propagation. Moreover, the jets used to protect the conductors of the focusing elements should be as close to the beams as possible to minimize the flux of neutrons entering the beam ports. It has not proved easy to make smooth, high-velocity jets, particularly if they are oscillating. Peterson and his collaborators at UC Berkeley have recently produced smooth, oscillating jets of water scaled hydrodynamically to be similar to jets of lithium containing fluids that might be used in a power plant [3]. There is also important research in liquid wall protection at UCLA [4] and Georgia Tech. [5]

Target injection and tracking are important technologies that must be developed for a power plant. Petzoldt built a simple air rifle capable of accelerating surrogate targets to a velocity of approximately $100 \mathrm{~m} / \mathrm{s}$. Photodiodes measure the transverse position of the target about $1 \mathrm{~m}$ beyond the end of the barrel. The position of the barrel and the measurement by the diodes are used to predict the target position in a chamber located approximately $2 \mathrm{~m}$ from the diodes. This prediction is compared to a measured position in the chamber, again made with photodiodes. The rifle itself was not particularly accurate. The measured position of the target in the chamber varies by several $\mathrm{mm}$; however, the rms difference between the predicted position and the measured position is less than 100 microns. This precision extrapolates to adequate precision for indirectly driven targets in a power plant where the injection distances are longer than the $2 \mathrm{~m}$ in Petzoldt's experiments. [6]

The Defense Inertial Confinement Fusion has always sponsored a substantial level of research in target fabrication. It is likely that some of the fabrication techniques that have been developed, e.g., beta layering and diffusion filling, will also be useful for fusion power production. However, power production imposes some additional constraints. The targets must be robust enough to survive the acceleration associated with injection. They must survive in the chamber environment. They must be made of materials that are inexpensive, environmentally acceptable, and chemically compatible with the rest of the chamber system. Finally, the targets must be mass produced at low cost.

Target survival is a particularly important issue for direct drive. If the target is injected too slowly, the high temperature in the reactor (usually $>500 \mathrm{~K}$ ) will destroy the integrity of the cryogenic fuel layer. If the target is injected too rapidly, the aerodynamic drag in the gas into the chamber will also produce too much heat. Calculations and experiments addressing this issue are in progress. Although indirectly driven targets are thermally protected by the hohlraum, they have more issues than directly driven targets in terms of materials that are environmentally and chemically acceptable. Some of the issues associated with target materials, fabrication, and injection were discussed at this Symposium [7]

Accelerators and Beam Physics. Prior to 1996, we had planned to build ILSE (Induction Linac Systems Experiment, later called Elise) [8]. Instead, we changed our plans and initiated a series of scaled experiments designed to address all systems and beam manipulations needed for a full-scale driver. We have now completed these experiments. We adopted the position that the experiments should be driver-like in terms of key 
dimensionless parameters such as phase advance $\left(\sigma_{0}\right)$ and depressed phase advance $(\sigma)$ but not driver-scale in parameters such as total ion kinetic energy or line charge density. Recent experiments have addressed transverse beam combining, ballistic focusing with and without neutralization, channel transport, and self-pinched propagation in the chamber. The scaled experiments are described in detail in these [9] and previous proceedings [1]. Briefly, these experiments have validated analytical and numerical predictions of beam behavior. They have served to benchmark our simulation tools, and, as noted in Section I, they have shown that it is possible to produce, transport, and accelerate beams having more than adequate brightness for fusion applications. Our injector research is the one major exception to the philosophy of scaled experiments. Our present 2-MV injector produces a single driverscale beam (line charge density of the order of $10^{-7} \mathrm{C} / \mathrm{m}$ or 300 to $800 \mathrm{~mA}$ of potassium or cesium).

Although the experiments have generally validated our theoretical expectations, they have also uncovered some areas that need additional research. For example, the reliability and lifetime of our ion sources require improvement. The injector beam optics must also be improved, and it would be desirable to reduce the cost and size of our injector systems. In addition, we believe that it is necessary to perform new experiments in acceleration, transport and focusing of beams, having driver-scale line charge density, before proceeding to the IRE. Our plans to perform such experiments are described in Section III.

Our theoretical goal is source-to-target simulation of the entire accelerator. We are using and developing three classes of computer codes. (1) Particle-in-cell codes. Our main code for studying beam dynamics in the accelerator itself is WARP, a 3-dimensional code with a detailed description of the lattice. For beam propagation in the target chamber, our main codes are the electromagnetic codes BIC, BPIC, and LSP. The first two have been developed primarily at Berkeley and Livermore while LSP has been developed by Mission Research Corporation. (2) Codes that evolve the particle distribution function (f). BEST, developed at Princeton, is a delta-f code that evolves perturbations around some $\mathrm{f}$ that is believed to be approximately correct. SLV (Semi-Lagrangian Vlasov) evolves f on a 4dimensional grid (two spatial dimensions and two momenta). This code has proved to be particularly useful for studying processes such as beam halo formation because, unlike the particle-in-cell codes, it is not limited by the statistics of small numbers in regions where there are few particles. (3) Codes that follow moments of $\mathrm{f}$. These codes run relatively rapidly and are used for synthesis of lattices and waveforms. CIRCE, a code originally developed at LLNL to study induction recirculators is one example of this class of codes.

The status of the codes and some recent results are given in the paper by Friedman [10]. While there has been significant progress since the last Symposium, much work remains to be done before we can truly simulate the entire accelerator. Current code development efforts focus on improved models for cavity impedance and the beam-beam interaction in multi-beam accelerators.

\section{Plans}

As described in Section I, we have just completed the Concept Exploration phase of the program and are beginning the Proof of Principle phase. The goals of each phase of the program are determined by working backward from the ultimate goal of an IFE Demo. The goals of the PoP phase of the program, according to this principle, are determined by the goals of the Performance Extension phase. The accelerator research part of Performance Extension is the IRE. Consequently, before describing our near-term PoP goals, we give a brief description of the IRE goals. 
The IRE must address a wide variety of scientific issues. Some of these issues are associated with high intensity beam physics in the accelerator itself. Here the IRE must determine and, if possible, expand the limits on long-term transverse and longitudinal beam dynamics. It must measure transverse and longitudinal emittance growth. For example it must determine under what conditions there are longitudinal instabilities. If these instabilities are deleterious, it must provide a vehicle to develop countermeasures, e.g., feedback stabilization. The IRE must allow us to study halo formation and its effect on required beam clearance. It must allow us to study effects associated with high intensity beams such as the presence of unwanted electrons.

There are also important issues in the target chamber. Most heavy ion fusion concepts have, in the past, been designed to operate at sufficiently high kinetic energy that beam neutralization in the chamber is not required. Operation at lower kinetic energy (actually lower voltage) leads to lower cost for induction linacs but requires beam neutralization. The IRE must be capable of validating the unneutralized propagation mode and it must be capable of definitive research on the neutralized propagation modes such as neutralized ballistic focusing and the various channel transport modes.

The IRE must be capable of addressing remaining target physics issues such as validating our understanding of the beam-plasma interaction. Additionally, it would be desirable to be able to study target physics issues such as fluid instabilities in direct drive and other topics in high energy density physics.

These general goals in accelerator physics, chamber propagation, focusing physics, and target physics lead to quantitative IRE requirements. For example, to study long-term transverse and longitudinal beam dynamics requires hundreds of lattice periods and enough beam current $(>100 \mathrm{~A}$ ) to load the induction modules and pulsers. It appears difficult to do definitive transport and focusing experiments at kinetic energies less than $100 \mathrm{MeV}$, at a perveance less than $10^{-4}$, and a normalized emittance larger than $5 \pi \mathrm{mm} \bullet \mathrm{mr}$. Our current criteria for studying the beam-plasma interaction are a beam plasma frequency and a plasma temperature within a factor of a few of the beam plasma frequency and temperature at the focus of a full-scale driver. These criteria require the IRE to have a focused intensity $>3 \mathrm{x}$ $10^{12} \mathrm{~W} / \mathrm{cm}^{2}$ and a total beam energy of several kilojoules - all within the cost goal shown in Figure 1. This cost goal corresponds to a total cost of the accelerator itself of approximately $\$ 150 \mathrm{M}$. It is not possible, at the present time, to build such a machine at this price so technology improvement is an important part of the PoP phase of the program.

We are exploring IRE options producing from 30 to $300 \mathrm{~kJ}$ of energy. One specific example produces $30 \mathrm{~kJ}$ of energy by accelerating $\mathrm{K}_{+}$ions to $200 \mathrm{MeV}$. This example has 32 beams and a length of approximately $300 \mathrm{~m}$. We have not yet performed a cost estimate for this design.

In order to be in a position to build the IRE, and to obtain the widespread support necessary to get such a project approved, we have adopted a number of aggressive PoP research goals:

1. Source-to-target simulation of full-scale drivers and the IRE.

2. Development of driver-scale ion sources and injectors. As noted above, we must improve the reliability and optics of our injection systems. Therefore one new experiment involves the design and construction of a high-current injector module. We are investigating two different approaches. The first uses large diameter, low current density thermionic sources (hot plate or alumino-silicate sources). These sources have been our main line for many years. They produce a current of the order of 1 A per beam. They can produce low emittance beams but they are large, have limited life, and have high power consumption. Size is a particular issue for systems that require many 
beams (10 to 1000). Consequently we are developing a second approach that uses hundreds of miniature high current density beamlets to form a single beam. Presently, both plasma sources and arc sources are candidates. The injector designs using the multiple beamlet approach are much more compact than the designs using thermionic sources. There are, however, important issues associated with gas loading and its effect on energy spread and voltage holding capability. We are building a new $500 \mathrm{kV}$ ion source test stand at Livermore to address these issues. The paper by Kwan et al. [11] gives more details about the injector experiments.

3. A High Current Experiment (HCX). Here the goal is to transport driver scale current (approximately $1 \mathrm{~A} / \mathrm{beam}$ ) through electrostatic and magnetic lattices (10 to 100 lattice periods). The HCX will extend our data base by nearly two orders of magnitude in current. The paper by Seidl [9] gives more details.

4. Technology development. The main cost centers of an induction linac for inertial fusion are multi-beam quadrupole arrays, insulators, ferromagnetic materials for the induction cores, and pulsers. We are developing electrostatic arrays and superconducting and normal magnetic arrays. For the magnetic arrays our goal is an array cost of $<\$ 10 / \mathrm{kA} \bullet \mathrm{m}$ of conductor. We have a contract with Advanced Magnet Laboratory to develop low-cost fabrication techniques. The first superconducting test coils are now being tested at MIT. The insulator cost goal is $\langle \$ 0.01 / \mathrm{V}$. One possibility for achieving this goal is to cast glassy ceramic insulators with imbedded metal rings for grading and for flanges. Advanced Ceramics has just delivered our first test insulators. They have a diameter of approximately half a meter. Our cost goal for ferromagnetic materials is $\$ 5$ to $\$ 10 / \mathrm{kg}$. Commercial materials meeting this cost goal are available for low frequency applications in the power industry. We are attempting to adapt these materials to pulsed applications by annealing and developing appropriate interlaminar insulation. There has been good progress as described in the paper by Molvik et al. [12]. The cost goal for pulsers is $\left\langle \$ 10^{-5} / \mathrm{W}\right.$ and $<\$ 20 / \mathrm{J}$. Additional research is needed in this area.

If we are successful in the four areas outlined above we will have a strong scientific and technical basis for the IRE.

\section{Summary}

In summary, the U.S. Department of Energy has established a new, larger inertial fusion energy program. The program has a new road map with clear milestones and cost goals leading to a demonstration power plant. We have established a Virtual National Laboratory for Heavy Ion Fusion and a Virtual Laboratory for Technology to integrate the research programs at different institutions. Progress in target design has reduced the beam energy requirements by more than a factor of two in the last several years. There have been important experiments in chamber dynamics and other inertial fusion technologies. The accelerator program is moving from a Concept Exploration phase to a Proof of Principle phase. Finally, there has been important progress toward a new accelerator facility, the Integrated Research Experiment or IRE.

The author thanks all the researchers from the VNL, from other laboratories, and from industry who have contributed to the research outlined in this paper. He also thanks Lynn Heimbucher for her help in preparing the manuscript.

\section{$\underline{\text { References }}$}

[1] The proceeding of the previous symposia in this series describe much of the earlier research in this field. See for example: 
Proceedings of the $13^{\text {th }}$ International Symposium on Heavy Ion Inertial Fusion, Nucl. Intsr. Meth. 278 (1998).

Proceedings of the Seventh International Symposium on Heavy Ion Inertial Fusion, Fus. Eng. Des. 32-33 (1996).

Proceedings of the $12^{\text {th }}$ International Symposium on Heavy Ion Inertial Fusion, Nucl. Instr. Meth. A415 (1998).

[2] Callahan-Miller, D.A., et al., "Progress in Target Physics and Design for Heavy Ion Fusion" Phys. Plasmas 7 (2000) 2083 and Callahan-Miller, D.A., et al., "Progress in Heavy Ion Fusion Targets" Proceedings of the13th International Heavy Ion Fusion Symposium. To be published in Nucl. Instr. Meth. and private communication.

[3] Peterson, P. "HIF Liquid Hydraulics Scaling and Pocket Design" Proceedings of the13th International Heavy Ion Fusion Symposium. To be published in Nucl. Instr. Meth.

[4] Morley, N.B., et al., "Experimental Investigation of Free Liquid Metal Jets in Vacuum: Preliminary results for IFE Chamber Wall Protection Applications", Fusion Technology, 34 (1998) 1035 and Konkachbaev, A., et al., "Stability and Contraction of a Rectangular Liquid Metal Jet in Vacuum Environment", to appear in Fusion Engineering and Design, 2000.

[5] Elwell, L.C., et al., "Dynamics of Obliquely Oscillated Turbulent Free Rectangular Jets", Bulletin of the American Physical Society 44 (8).

[6] Petzoldt, R.W. "IFE Target Injection and Tracking Experiment", Fusion Technology 34 (1998) 831.

[7] Latkowski, J.F., et al., "Selection of IFE target materials from a safety and environmental perspective" Proceedings of the13th International Heavy Ion Fusion Symposium. To be published in Nucl. Instr. Meth.

Schultz, K.R., et al., "IFE Target Fabrication and Injection - Achieving "Believability" Proceedings of the13th International Heavy Ion Fusion Symposium. To be published in Nucl. Instr. Meth.

[8] Kwan, J., "Elise Plans and Progress", Fusion Eng. Des. 32-33 (1996) 299-308

[9] Seidl, P.A., et al., "Experiments at the Virtual National Laboratory for Heavy Ion Fusion", MacLaren, S.A., et al., "Results from the Scaled Final Focus Experiment", and Ponce, D.M., et. al., "Diagnostics of Plasma Channel for HIF Transport", Proceedings of the13th International Heavy Ion Fusion Symposium. To be published in Nucl. Instr. Meth.

[10] Friedman, A. et al., "Beam Simulations for IRE and Driver-status and Strategy", Proceedings of the 13th International Heavy Ion Fusion Symposium. To be published in Nucl. Instr. Meth.

[11] Kwan, J., et al.,"Ion Sources and Injectors for HIF Induction Linacs", Proceedings of the 13th International Heavy Ion Fusion Symposium. To be published in Nucl. Instr. Meth.

[12] Molvik, A., et al., "Induction Accelerator Efficiency at $5 \mathrm{~Hz}$ ", Proceedings of the13th International Heavy Ion Fusion Symposium. To be published in Nucl. Instr. Meth.

This work was supported by the Director, Office of Science, Office of Fusion Energy Sciences, of the U.S. Department of Energy under Contract No. DE-AC03-76SF00098 at the University of California Lawrence Berkeley National Laboratory. 